\title{
Avaliação da produção de óxido nítrico em ratos, submetidos aos exercícios aeróbio e anaeróbio
}

\author{
Vanessa Guimarães de Freitas Cruvelo D'Ávila ${ }^{*}$, Nozelmar Borges de Sousa Júnior², Fábio \\ Borges de Sousa ${ }^{3}$, Lídia Andreu Guillo ${ }^{1}$ \\ ${ }^{1}$ Departamento de Bioquímica e Biologia Molecular, Instituto de Ciências Biológicas, Universidade Federal de \\ Goiás, ${ }^{2}$ Departamento de Desporto e Reabilitação, Universidade Salgado de Oliveira, ${ }^{3}$ Departamento de Ciências \\ Fisiológicas, Instituto de Ciências Biológicas, Universidade Federal de Goiás
}

*Correspondência:

V. G. F. C D’Ávila

Departamento de Bioquímica e

Biologia Molecular

Instituto de Ciências Biológicas Universidade Federal de Goiás -UFG

Campus II -Jardim Samambaia

74001-970 - Goiânia - GO, Brasil

E-mail: vanessa@fmb.edu.br
Óxido nítrico (NO) exerce influências muito importantes em vários processos fisiológicos. Neste trabalho avaliamos a produção de NO sanguíneo em ratos Wistar, submetidos ao nado aeróbio e anaeróbio agudos. A formação do óxido nítrico foi verificada através da dosagem dos produtos de oxidação estáveis do metabolismo do óxido nítrico (nitratos). Para isso utilizamos o método colorimétrico de Griess. Verificamos a existência de uma diferença significativa ( $p=0,000261)$ na produção de óxido nítrico entre a realização do nado aeróbio e o anaeróbio, na qual o aeróbio mostrou-se mais eficiente na promoção de níveis mais elevados. O exercício aeróbio agudo com duração de no mínimo 10 minutos mostrou-se mais eficaz no quesito produção de NO em relação ao exercício de 5 minutos. A positiva relação observada entre o exercício aeróbio e a formação de NO pode ajudar a explicar os efeitos benéficos do exercício na saúde cardiovascular. Sabemos que a prática de exercício aeróbio e sua duração aumentam a biodisponibilidade de NO, o qual é considerado importante regulador fisiológico da pressão arterial.
Unitermos

- Óxido nítrico/efeito fisiológico

- Reação de Griess

- Exercícios Físicos/estudo experimental

- Hipertensão Arterial/ regulação

\section{INTRODUÇÃO}

O gás simples, óxido nítrico (NO), controla uma variedade de processos biológicos complexos, incluindo a homeostase da pressão sangüínea, agregação plaquetária e transmissão de sinais pelo sistema nervoso, sendo também, importante para a função do sistema imune, desempenhando papel chave na atividade de macrófagos e defesas celulares contra patógenos microbianos (Durner et al, 1999). Um aspecto marcante desta molécula é a sua capacidade de ser benéfica ou potencialmente tóxica dependendo da concentração ou depuração tecidual (Flora Filho, Zilberstein, 2000).
Várias pesquisas afirmam que o exercício físico está associado à estimulação da produção de NO (Higashi, Yoshizumi, 2004; Jungersten et al., 1997; Croix et al., 1999). Estudos experimentais em animais e humanos têm sugerido que aumentos na produção de óxido nítrico podem contribuir para a hipotensão observada após exercício aeróbico (Halliwill et al., 2000; Jungersten et al., 1997). Da mesma forma, observou-se aumento drástico da pressão arterial (PA) em modelos animais submetidos à inibição da produção endógena de óxido nítrico (Nava, Luscher, 1995).

No presente estudo, procurou-se comparar os níveis de óxido nítrico plasmático de ratos Wistar submetidos ao 
exercício físico aeróbio e anaeróbio. Apesar das limitações deste estudo experimental com animais, pretendemos contribuir com as discussões sobre o tipo de atividade física mais eficiente para a elevação dos níveis plasmáticos de óxido nítrico, visando à seleção de programas de treinamento mais adequados nas situações fisiopatológicas ou mesmo para a manutenção de vida saudável.

\section{MATERIAL E MÉTODOS}

\section{Animais}

Para o desenvolvimento deste estudo foram utilizados 25 ratos machos adultos (aproximadamente 90 - 100 dias) Wistar (Rattus novergicus albinus, Wistar). Os animais provenientes do Biotério Central da Universidade Federal de Goiás (UFG) foram mantidos no Laboratório de Neurobiologia do Departamento de Morfologia do Instituto de Ciências Biológicas da UFG. Foram alojados em gaiolas coletivas (com três ratos por gaiola) à temperatura ambiente de $25{ }^{\circ} \mathrm{C}$ e obedecendo a um fotoperíodo de 12 horas claro / 12 horas escuro, recebendo ração comercial Labina / Purina ${ }^{\circledR}$ e água Milli Q (ultra-pura) à vontade. O projeto desenvolvido foi aprovado pelo Comitê de Ética em Pesquisa Médica Humana e Animal do Hospital das Clínicas da UFG (protocolo CEPMHA/HC/UFG n ${ }^{\circ} 103 / 04$ ).

Foram formados cinco blocos, constituídos cada um por cinco animais, os quais foram submetidos aos cinco tratamentos distintos, como segue:

Blocos - Grupo Controle, Repouso (GC);

Exercício Aeróbio Agudo no estágio um (EAA); Exercício Aeróbio Agudo no estágio dois $\left(\mathrm{EAA}_{2}\right)$; Exercício Aeróbio Agudo no estágio três $\left(\mathrm{EAA}_{3}\right)$; Exercício Anaeróbio Agudo (EAnA).

\section{Exercícios físicos agudos}

Com exceção do GC, os animais dos demais grupos foram submetidos a sistema de natação, no qual a piscina utilizada tinha como medidas $60 \mathrm{~cm}$ de profundidade e 50 $\mathrm{cm}$ de diâmetro, com água aquecida por sistema de resistência elétrica a $31 \pm 2^{\circ} \mathrm{C}$.

Os animais (exceto $\mathrm{GC}$ ) passaram por um período de adaptação ao meio líquido que consistia em deixar os animais durante 60 minutos diários por duas semanas, em contato com água rasa. Esta fase de adaptação foi realizada para evitar a ocorrência de estresse intenso, advindo do medo natural que os animais têm normalmente de água e de profundidade (Harri, Kuusela, 1986).

Após a adaptação, todos os animais foram pesados. À base da cauda de cada rato do grupo $\mathrm{EAA}_{1}$ foi atado um peso (chumbos) que correspondia a $1 \%$ de seus pesos corporais. Estes animais se exercitaram com a carga por 5 minutos. Em seguida, foi retirada uma alíquota de sangue para a dosagem de lactato e de nitrato sanguíneos. No estágio EAA2, os animais nadaram com a mesma carga de $1 \%$ de seus pesos corporais durante 10 minutos, sendo retirada uma alíquota de sangue para dosagem de nitrato e lactato. No estágio EAA3, o nado era realizado com a mesma carga de $1 \%$ do peso corporal durante 20 minutos e nova retirada de sangue foi executada para dosagem de nitrato e lactato. A metodologia para o nado aeróbio agudo foi esquematizada a partir de estudos já realizados por outros autores (Dos Santos, De Mello, 2003; Afonso et al., 2003; Voltarelli et al., 2004; Voltarelli et al., 2002; Gobatto et al., 2001; Prada et al., 2004).

Os animais do grupo EAnA executaram o nado de forma progressiva, no qual as cargas variavam em escala de $1 \%$ do peso corporal de cada animal, iniciando-se com $2 \%$ e aumentando-se até $8 \%$ do peso corporal. O tempo de duração total do exercício foi de 27 minutos, sendo a duração do nado com cada carga de três minutos com pausa de um minuto entre as séries para troca dos pesos (Dos Santos, De Mello, 2003). Logo em seguida, foi retirada uma alíquota sanguínea para subseqüente dosagem da concentração de lactato e nitrato.

\section{Obtenção do material biológico}

Alíquotas de sangue dos ratos dos grupos GC, EAnA, EAA EAA $_{2}$ e EAA foram retiradas através de punção intra-cardíaca, utilizando-se agulhas Nipro $^{\circledR}(30 /$ 7), seringas $\mathrm{BD}^{\circledR}(5 \mathrm{~mL})$ e tubos coletores estéreis Vacuum II $^{\circledR}(10 \mathrm{~mL})$, sem heparina.

Imediatamente após a coleta, o sangue foi centrifugado a $3000 \mathrm{rpm}$ por 2 minutos e o soro transferido para tubos eppendorf previamente lavados com água Milli-Q e autoclavados. Para desproteinização da amostra, utilizou-se mistura de metanol e éter $(3: 1, \mathrm{v} / \mathrm{v})$, adicionada em quantidades iguais ao volume de soro sangüíneo, segundo Guevara et al. (1998), com algumas modificações. A solução foi mantida à temperatura ambiente por 10 minutos e logo após este período foi centrifugada por $10 \mathrm{minu}$ tos a $10.000 \mathrm{rpm} \mathrm{a} 4{ }^{\circ} \mathrm{C}$. O sobrenadante obtido deste procedimento foi armazenado em tubos eppendorf à temperatura de $4{ }^{\circ} \mathrm{C}$, para análise de nitrato plasmático.

A alíquota de sangue necessária para a dosagem do lactato dos animais dos grupos EAnA e $\mathrm{EAA}_{1}, \mathrm{EAA}_{2} \mathrm{e}$ $\mathrm{EAA}_{3}$ foi obtida através de corte na região distal da cauda de cada rato, coletando-se quantidade suficiente para preencher a região da fita teste (amarela) do analisador de lactato Accutrend ${ }^{\circledR}$ Lactate (Roche Diagnóstica Brasil). 


\section{Dosagem de nitrato plasmático}

A partir de adaptação na metodologia para a dosagem de Nitrato (Miranda et al., 2001), no presente estudo o procedimento consistiu da adição em tubos eppendorf $(1,5 \mathrm{~mL})$ de $200 \mu \mathrm{L}$ de soro sangüíneo desproteinizado, $100 \mu \mathrm{L} \mathrm{de} \mathrm{VCl}_{3}$ (cloreto de vanádio III), $150 \mu \mathrm{L}$ de Sulfanilamida $2 \%$ e $50 \mu \mathrm{L}$ de NEED $0,1 \%$ (naftiletilenodiamino Dihidroclorídrico 0,1\%). Sendo assim, os tubos foram homogeneizados em vórtex por 10 segundos, centrifugados por 60 segundos a $10.000 \mathrm{rpm}$ e em seguida incubados em banho-maria à temperatura de $37^{\circ} \mathrm{C}$ por 60 minutos. Logo após nova homogeneização e centrifugação durante 60 segundos em $12.000 \mathrm{rpm}$, transferiu-se $300 \mu \mathrm{L}$ de cada amostra para uma placa de 96 poços para leitura da absorbância a $540 \mathrm{~nm}$ em espectrofotômetro, ELx 800 Universal Microplate Reader (Bio-TEK Instruments, San Diego, USA).

\section{Curva padrão de nitrato de sódio}

As soluções padrão de nitrato de sódio foram preparadas a partir de solução-estoque de $0,05 \mu \mathrm{mol} / \mathrm{L}$. As concentrações iniciais abrangiam a faixa de $50 \mu \mathrm{mol} / \mathrm{L}$ a 500 $\mu \mathrm{mol} / \mathrm{L}$ ( 5 soluções). O preparo destas soluções foi realizado em fluxo laminar e utilizaram-se materiais previamente lavados com água Milli-Q e autoclavados.

A curva padrão de nitrato de sódio foi construída a partir da adição de $20 \mu \mathrm{L}$ de cada solução padrão preparada a $180 \mu \mathrm{L}$ de água Milli-Q. Logo em seguida, adicionouse $100 \mu \mathrm{L}$ de cloreto de vanádio e $200 \mu \mathrm{L}$ de uma mistura de sulfanilamida e naftiletilenodiamino. O procedimento foi realizado em tubos Eppendorf $(1,5 \mu \mathrm{L})$, sempre mantidos em gelo. A leitura das amostras sanguíneas era sempre realizada em conjunto com as soluções-padrão.

\section{Dosagem de lactato sanguíneo}

Para a dosagem do lactato sanguíneo, utilizamos o aparelho Accutrend ${ }^{\circledR}$ Lactate cuja tira teste possui uma zona de teste contendo reagentes de detecção.

\section{Análise estatística}

Os dados são apresentados através da média \pm desvio padrão de cinco animais em cada bloco ou treinamento. Para comparação dos resultados obtidos, através de Delineamento em Blocos Casualizados, usamos o método de Análise de Variância (ANOVA) e, posteriormente, o teste de significância Fisher's Least Significant Difference (LSD/Fisher). Utilizou-se o software STATISTICA versão 6.0 para anali- sar os valores de lactato e nitrato sangüíneos encontrados nos grupos GC, EAA1, EAA2, EAA3 e EAnA.

\section{RESULTADOS}

\section{Lactato sangüíneo}

Observam-se, na Tabela I, os valores da predominância metabólica obtidos dos animais submetidos aos tratamentos EAA1; EAA2; EAA3. As respectivas médias fo$\mathrm{ram}: 3,56 \mathrm{mmol} / \mathrm{L} \pm 0,67,4,28 \mathrm{mmol} / \mathrm{L} \pm 0,24 \mathrm{e}$ $4,86 \mathrm{mmol} / \mathrm{L} \pm 0,93$. Estas se mantiveram abaixo do limiar anaeróbio de 7,17 $\pm 0,16 \mathrm{mmo} / \mathrm{L}$ descrito por outros autores para esses animais (Voltarelli et al., 2002). Observamos que a média obtida a partir dos valores do grupo EAnA foi de $9,2 \pm 0,43 \mathrm{mmol} / \mathrm{L}$, superou o valor do limiar anaeróbio.

TABELA I - Efeito dos diferentes tratamentos nos níveis de lactato sangüíneo. A concentração de lactato sanguíneo foi determinada imediatamente após a realização dos exercícios aos quais os ratos foram submetidos: EAA1 (exercício aeróbio agudo por 5 minutos); EAA2 (exercício aeróbio agudo por 10 minutos); EEA3 (exercício aeróbio agudo por 20 minutos) e EAnA (exercício anaeróbio) e GC (sedentário, grupo controle). A análise ANOVA demonstrou que os tratamentos diferem significativamente entre $\operatorname{si}(\mathrm{p}<0,0001)$

\begin{tabular}{lcccc}
\hline $\begin{array}{l}\text { Blocos de } \\
\text { Animais }\end{array}$ & $\begin{array}{c}\text { EAA1 } \\
(\mathrm{mmol} / \mathrm{L})\end{array}$ & $\begin{array}{c}\text { EAA2 } \\
(\mathrm{mmol} / \mathrm{L})\end{array}$ & $\begin{array}{c}\text { EAA3 } \\
(\mathrm{mmol} / \mathrm{L})\end{array}$ & $\begin{array}{c}\text { EAnA } \\
(\mathrm{mmol} / \mathrm{L})\end{array}$ \\
\hline $1^{\circ}$ & 2,8 & 4,4 & 6,2 & 9,4 \\
$2^{\mathrm{o}}$ & 3,9 & 4,6 & 4,8 & 9,1 \\
$3^{\circ}$ & 4,5 & 4,0 & 4,1 & 8,5 \\
$4^{\text {o }}$ & 3,1 & 4,3 & 3,9 & 9,4 \\
$5^{\text {o }}$ & 3,5 & 4,1 & 5,3 & 9,6 \\
\hline $\begin{array}{l}\text { Média } \pm \\
\text { Desvio }\end{array}$ & $3,56 \pm 0,67$ & $4,28 \pm 0,24$ & $4,86 \pm 0,93$ & $9,2 \pm 0,43$ \\
Padrão & & & & \\
\hline
\end{tabular}

A dosagem deste metabólito foi realizada para confirmar a intensidade real em que cada animal estava se exercitando. Concentrações abaixo ou acima do limiar caracterizam o exercício como aeróbio e anaeróbio, respectivamente, validando nossa abordagem experimental.

\section{Nitrato de sódio sangüíneo}

Com a construção da curva-padrão, obtivemos o coeficiente de regressão e a equação da reta, a qual nos permitiu determinar a concentração de $\mathrm{NO}$ em $\mu \mathrm{mol} / \mathrm{L}$ a 
TABELA II - Efeito dos diferentes tratamentos nos níveis plasmáticos de nitrato. A concentração de nitrato/nitrito plasmático foi determinada segundo metodologia descrita em Material e Métodos, após a realização dos exercícios aos quais os ratos foram submetidos: GC (grupo controle, repouso); EAA1 (exercício aeróbio agudo por 5 minutos); EAA2 (exercício aeróbio agudo por 10 minutos); EEA3 (exercício aeróbio agudo por 20 minutos) e EAnA (exercício anaeróbio). A análise ANOVA demonstrou que os tratamentos diferem significativamente entre si $(p=0,000261)$

\begin{tabular}{lccccc}
\hline Blocos de Animais & $\begin{array}{c}\text { GC } \\
(\mu \mathrm{mol} / \mathrm{L})\end{array}$ & $\begin{array}{c}\text { EAA1 } \\
(\mu \mathrm{mol} / \mathrm{L})\end{array}$ & $\begin{array}{c}\text { EAA2 } \\
(\mu \mathrm{mol} / \mathrm{L})\end{array}$ & $\begin{array}{c}\text { EAA3 } \\
(\mu \mathrm{mol} / \mathrm{L})\end{array}$ & $\begin{array}{c}\text { EAnA } \\
(\mu \mathrm{mol} / \mathrm{L})\end{array}$ \\
\hline $1^{\mathrm{o}}$ & 4,12 & 8,04 & 15,35 & 15 & 7,38 \\
$2^{\mathrm{o}}$ & 3,48 & 8,52 & 10,25 & 13,06 & 3,83 \\
$3^{\mathrm{o}}$ & 4,48 & 6,14 & 14,4 & 8,93 & 4,48 \\
$4^{\mathrm{o}}$ & 4,71 & 6 & 8,42 & 8,98 & 9,53 \\
$5^{\mathrm{o}}$ & 3,22 & 8,26 & 7,86 & 9,21 & 3,88
\end{tabular}

\begin{tabular}{llllll}
\hline Média \pm Desvio Padrão & $4,00 \pm 0,64$ & $7,39 \pm 1,22$ & $11,26 \pm 3,44$ & $11,04 \pm 2,82$ & $5,82 \pm 2,54$ \\
\hline
\end{tabular}

partir das absorbâncias obtidas. Na Tabela II, estão dispostos os valores destas concentrações.

Conforme observamos na Tabela II, os tratamentos diferem significativamente entre si e após a aplicação do teste de significância LSD / Fisher observamos aumento significativo $(p=0,028915 ; p=0,0001 ; p=0,000136)$ na produção de nitrato sanguíneo em ratos que foram submetidos aos exercícios aeróbios agudos (EAA1, EAA2 e EAA3, respectivamente) em comparação aos animais que permaneceram em repouso (GC) (ver Tabela III).

TABELA III - Valores de p obtidos após aplicação do Teste de significância LSD/Fisher aos resultados obtidos na Tabela II. * Diferença significativa $(\mathrm{p}<0,05)$

\begin{tabular}{lcccc}
\hline & EAA1 & EAA2 & EAA3 & EAnA \\
\hline GC & $0,028915^{*}$ & $0,000100^{*}$ & $0,000136^{*}$ & 0,216362 \\
EAA1 & & $0,014660^{*}$ & $0,020146^{*}$ & \\
EAA2 & & & 0,878173 & \\
\hline
\end{tabular}

Também pudemos observar que não houve diferença significativa $(p=0,216362)$ entre os valores de nitrato encontrados nos animais sedentários (GC) e os valores obtidos pelos ratos que se exercitaram anaerobiamente (EAnA). Da mesma forma, não houve diferença significativa $(p=0,878173)$ entre os valores de nitrato encontrados no sangue dos animais que realizaram exercícios aeróbios agudos, no estágio 2 e no estágio 3.

Por outro lado, observamos uma elevação significativa nos valores de nitrato no sangue dos ratos submetidos aos exercícios aeróbios agudos no estágio 2 e 3 em relação aos valores encontrados nos animais submetidos ao exercício aeróbio agudo no estágio $1(\mathrm{p}=0,014660 \mathrm{e}$ $\mathrm{p}=0,020146$, respectivamente).

\section{DISCUSSÃO}

O óxido nítrico é uma molécula importante para o controle metabólico durante o exercício, pois uma deficiência na sua produção, particularmente pelo sistema vascular endotelial, contribui para as limitações no treinamento físico associadas às doenças cardiovasculares (Kingwell, 2000).

Analisando-se os níveis de óxido nítrico encontrados nos grupos EAA1 (5 min), EAA2 (10 min) e EAA3 (20 min), observamos que os animais que realizaram o nado durante 10 minutos e 20 minutos apresentaram níveis significativamente mais elevados de NO em comparação aos valores obtidos pelos ratos que nadaram por 5 minutos. No entanto, não encontramos diferença significativa entre o nado com duração de 10 min e o nado de 20 min. Em humanos, observa-se que a duração do exercício influencia a magnitude da hipotensão pós-exercício, sendo que o exercício físico de maior duração provoca hipotensão pós-exercício maior e mais prolongada do que o exercício de curta duração (Forjaz et al., 1998). Nossos resultados com animais mostraram claramente a elevação dos níveis plasmáticos de óxido nítrico após uma sessão de $10 \mathrm{ou}$ 20 min de nado aeróbio.

Estudos epidemiológicos têm consistentemente revelado relação inversa entre atividade física (ou exercício) e a hipertensão arterial (PA), ou seja, o treinamento físico reduz significativamente a PA em pacientes com hipertensão arterial sistêmica (Lesniak, Dubbert, 2001). A alteração na função endotelial mediada pela redução da biodisponibilidade de NO está associada à hipertensão e a prática do exercício aeróbio tem demonstrado melhora na função endotelial em modelos animais hipertensos e em pacientes humanos com hipertensão essencial (Higashi, Yoshizumi, 2004). Em apenas uma sessão de exercício aeróbio verificou-se a promo- 
ção de efeito hipotensor em pacientes hipertensos (Rondon et al., 2002; Taylor-Tolbert et al., 2000). Sendo assim, o treinamento físico aeróbio tem sido recomendado no tratamento da hipertensão arterial por promover esta importante queda na PA (Forjaz et al., 2003).

Em nossos dados, não encontramos diferença significativa entre os níveis de $\mathrm{NO}$ de animais que realizaram exercício anaeróbio agudo (EAnA) e os níveis encontrados nos ratos que foram mantidos sedentários (GC). Possivelmente esta produção de NO, equivalente a valores basais, seja um dos fatores pelo qual o exercício anaeróbio não é usualmente recomendado a hipertensos.

Os exercícios anaeróbios apresentam efeitos cardiovasculares diferentes em função de sua intensidade. Assim, os exercícios de baixa intensidade que promovem melhorias na Resistência Muscular Localizada (RML), causam aumentos modestos da pressão durante sua execução, promovendo pequena queda da pressão arterial em hipertensos em longo prazo. Por outro lado, exercícios anaeróbios de alta intensidade, que visam à melhora da força/hipertrofia muscular, promovem aumento da pressão arterial durante sua execução, podendo intensificar aneurismas cerebrais preexistentes, que são mais comuns em hipertensos. Sendo assim, os exercícios anaeróbios de alta intensidade devem ser evitados em virtude da ausência do efeito hipotensor em longo prazo e do risco potencial durante a execução desse exercício (Forjaz et al., 2003).

Com base nos dados coletados neste trabalho, verificamos que existe diferença significativa na produção de óxido nítrico entre a realização do nado aeróbio e o anaeróbio, na qual o aeróbio mostrou-se mais eficiente na promoção de níveis mais elevados de óxido nítrico. Verificamos também que a duração total do treinamento anaeróbio (27 minutos) foi adequada para o estabelecimento da condição anaeróbica. Tendo em vista que o papel do NO na redução da pressão arterial durante exercícios anaeróbios em humanos ainda não está completamente resolvido, os resultados aqui encontrados sobre a produção de óxido nítrico em anaerobiose, contribuem para a compreensão da importância de exercícios físicos adequados em humanos. Além disso, a utilização de metodologia adequada ao se utilizar animais é de suma importância, uma vez que questões sobre esquemas ideais de treinamento ou mesmo a duração dos benefícios em humanos ainda permanecem sem resposta.

A positiva relação observada entre o exercício físico aeróbio e a formação de óxido nítrico plasmático em ratos, pode ajudar a explicar os efeitos benéficos do exercício na saúde cardiovascular. Segundo Kingwell (2000), a prática do exercício físico em indivíduos com risco cardiovascular elevado aumenta a biodisponibilidade de NO. Assim, pode ser sugerido às pessoas portadoras de hipertensão arterial que optem pela prática de exercícios aeróbios, tanto agudos quanto crônicos, adotando-os como conduta não-farmacológica no tratamento da hipertensão arterial. Vale lembrar, portanto, que a duração da sessão tem influência sobre o efeito hipotensor pós-exercício, daí a necessidade do acompanhamento por profissional da área.

\section{ABSTRACT}

\section{Evaluation of the production of nitric oxide in mice, submitted to aerobic and anaerobic exercises}

Nitric Oxide (NO) exerts important influences in several physiological processes. In this work we evaluated the production of sanguine NO in Wistar rats, submitted to the acute aerobic and anaerobic exercises. The formation of nitric oxide was verified through the dosage of the end products of oxidation of the metabolism of nitric oxide (nitrates). For this we used the colorimetric Griess method. We verified the existence of a significant difference ( $p=0.000261)$ in the production of NO among the accomplishment of the aerobic swimming and the anaerobic, where the aerobic was shown more efficient in the promotion of higher levels. The acute aerobic exercise with duration of at least 10 minutes was shown more effective in the requirement production of $N O$ in relation to the 5 minutes exercise. The positive relationship observed between the aerobic exercise and the formation of NO can help to explain the beneficial effects of the exercise in the cardiovascular health. We know that the practice of aerobic exercise and your duration increases the biodisponibility of NO, which is an important physiologic regulator of the blood pressure.

UNITERMS: Sodium nitrate. Griess Reaction. Physical exercises/experimental study. Arterial hypertension.

\section{AGRADECIMENTOS}

Os autores agradecem à Coordenação de Aperfeiçoamento de Pessoal de Nível Superior (CAPES), pela bolsa concedida à Vanessa Guimarães de Freitas Cruvelo D'Ávila. Nossos sinceros agradecimentos à Julita Danaso Santos pelo apoio técnico na punção cardíaca necessária para o progresso desta pesquisa, ao $\operatorname{Prof}^{0}$ M.Sc. Anderson Miguel da Cruz que generosamente dividiu seus conhecimentos e experiências e à FUNAPE/UFG e PROAD/UFG pelo auxílio financeiro. 


\section{REFERÊNCIAS BIBLIOGRÁFICAS}

AFONSO, M.; ZAGATTO, A. M.; DE SOUZA, C. N.; LUCIANO, E. Efeitos do exercício agudo de natação sobre as respostas metabólicas em ratos Wistar. Revista Motriz, v.9, p.S41-S108, 2003.

BERGHOLM, R.; MAKIMATTILA, S.; VALKONEN, M.; LIU, M. L.; LAHDENPERA, S.; TASKINEN, M. R.; SOVIJÄRVI, A.; MALMBERG, P.; YKI-JÄRVINEN, $\mathrm{H}$. Intense physical training decreases circulating antioxidants and endothelium-dependent vasodilation in vivo. Atherosclerosis, v.145, n.2, p.341-349, 1999.

CROIX, C. M. S. T.; WETTER, T. J.; PEGELOW, F. F.; MEYER, K. C.; DEMPSEY, J. A. Assessment of Nitric Oxide Formation During Exercise. Am. J. Respir. Crit. Care Med., v.159, p.1125-1133, 1999.

DOS SANTOS, J. W.; DE MELLO, M. A. R.; Reprodutibilidade do limiar anaeróbio de lactato em exercício de natação com ratos. Revista Motriz, v.9, n.1, p.S41-S108, 2003.

DURNER, J.; GOW, A. J.; STAMLER, J. S.; GLAZEBROOK, J.; Ancient origins of nitric oxide signaling in biological systems. PNAS, v.96, n.14, p.206207, 1999.

FLORA FILHO, R.; ZILBERSTEIN, B. Óxido Nítrico: O Simples mensageiro percorrendo a complexidade. Metabolismo, síntese e funções. Rev. Assoc. Med. Bras., v.46, p.265-271, 2000.

FORJAZ, C. L. M.; REZK, C. C.; DE MELO, C. M.; DOS SANTOS, D. A.; TEIXEIRA, L.; NERY, S. S.; TINUCCI, T. Exercício resistido para o paciente hipertenso: indicação ou contra-indicação. Rev. Bras. Hipertens., v.10, n.2, p.119-124, 2003.

FORJAZ, C. L. M.; SANTAELLA, D. F.; REZENDE, L. O.; BARRETTO, A. C. P.; NEGRÃO, C. E. A duração do exercício determina a magnitude e a duração da hipotensão pós-exercício. Arq. Bras. Cardiol., v.70, n.2, p.99-104, 1998.

GOBATTO, C. A.; DE MELLO, M. A. R.; SIBUYA, C. Y.; DE AZEVEDO, J. R. M.; DOS SANTOS, L. A.; KOKUBUN, E. Maximal lactate steady state in rats submitted to swimming exercise. Comp. Biochem. Physiol., v.130, p.21-27, 2001.
GUEVARA, I.; IWANEJKO, J.; DEMBINSKA-KIEC, A.; PANKIEWICZ, J.; WANAT, A.; ANNA, P.; GOL-BEK, I.; BARTU, S.; MALCZEWSKA-MALEC, M.; SZCZUDLIK, A. Determination of nitrite/nitrate in human biological material by the simple Griess reaction. Clin. Chim. Acta, v.274, n.2, p.177-188, 1998.

HALLIWILL, J. R.; MINSON, C. T.; JOYNER, M. J. Effect of Systemic Nitric Oxide Synthase Inhibition on Postexercise Hypotension in Humans. J. Appl. Physiol., v.89, p.1830-1836, 2000.

HARRI, M.; KUUSELA, P. Is swimming exercise or cold exposure for rats? Acta Physiol. Scand., v.126, p.189197, 1986.

HIGASHI, Y.; YOSHIZUMI, M,. Exercise and endothelial function: Role of endothelium-derived nitric oxide and oxidative stress in healthy subjects and hypertensive patients. Pharmacol. Ther., v.102, n.1, p.87-96, 2004.

JUNGERSTEN, L.; AMBRING, A.; WALL, B.; WENNMALM, A. Both physical fitness and acute exercise regulate nitric oxide formation in healthy humans. J. Appl. Physiol., v.82, p.760-764, 1997.

KINGWELL, B. A. Nitric oxide-mediated metabolic regulation during exercise: effects of training in health and cardiovascular disease. FASEB J., v.14, n.12, p.16851696, 2000 .

LESNIAK, K. T.; DUBBERT, P. M. Exercise and hypertension. Curr. Opin.Cardiol., v.16, p.356-359, 2001.

MIRANDA, K. M.; ESPEY, M. G.; WINK, D. A. A rapid, simple spectrophotometric method for simultaneous detection of nitrate and nitrite. Nitric Oxide, v.5, n.1, p.6271, 2001.

NATHAN, C. Nitric oxide as a secretory product of mammalian cells. FASEB J., v.6, n.12, p.3051-3064, 1992.

NAVA, E.; LUSCHER, T. F. Endothelium-derived vasoactive factors in hypertension: nitric oxide and endothelin. $J$. Hypertens. Suppl., v.13, p.S38-S48, 1995.

PRADA, F. J.A.; VOLTARELLI, F.A.; OLIVEIRA, C.A. M.; GOBATTO, C. A.; MACEDO, D. V.; MELLO, M.A.R. Condicionamento aeróbio e estresse oxidativo em ratos treinados por natação em intensidade equivalente ao limiar anaeróbio. Rev. Bras. Ciênc.Mov., v.12, p.29-34, 2004. 
RONDON, M. U. B.; ALVES, M. J.; BRAGA, A. M.; TEIXEIRA, O. T.; BARRETTO, A. C.; KRIEGER, E.M. Postexercise blood pressure reduction in eldely hypertensive patients. J. Am. Coll. Cardiol., v.39, p.676$682,2002$.

TAYLOR-TOLBERT, N. S.; DENGEL, D. R.; BROWN, M. D.; McCOLE, S. D.; PRATLEY, R. E.; FERREL, R. E.; HAGBERG, J. M. Ambulatory blood pressure after acute exercise in older men with essential hypertension. Am. J. Hypertens., v.13, n.1, p.44-51, 2000.
VOLTARELLI, F. A.; DE MELLO, M. A. R.; GOBATTO, C. A. Limiar anaeróbio determinado pelo teste de lactato mínimo em ratos: efeito dos estoques de glicogênio muscular e do treinamento físico. Rev. Port. Ciênc. Desp., v.4, p.16-25, 2004.

VOLTARELLI, F. A.; GOBATTO, C. A.; DE MELLO, M. A. R. Determination of anaerobic threshold in rats using the lactate minimum test. Braz. J. Med. Biol. Res., v.35, n.11, p.1389-1394, 2002.

Recebido para publicação em 21 de fevereiro de 2008 Aceito para publicação em 19 de agosto de 2008 